21.2

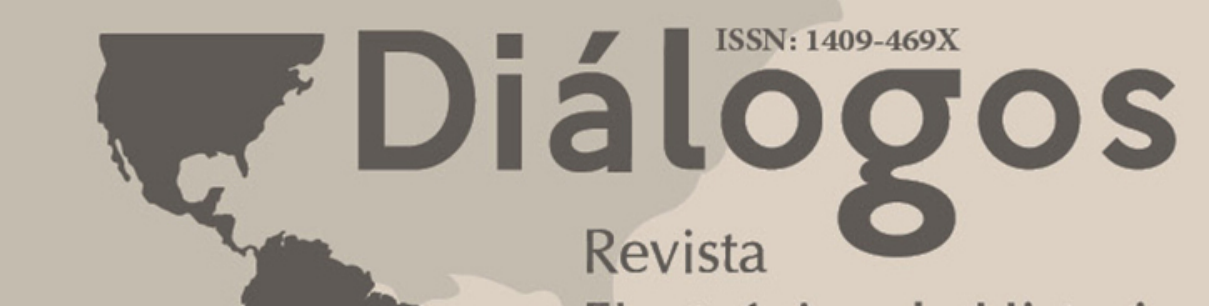

Electrónica de Historia

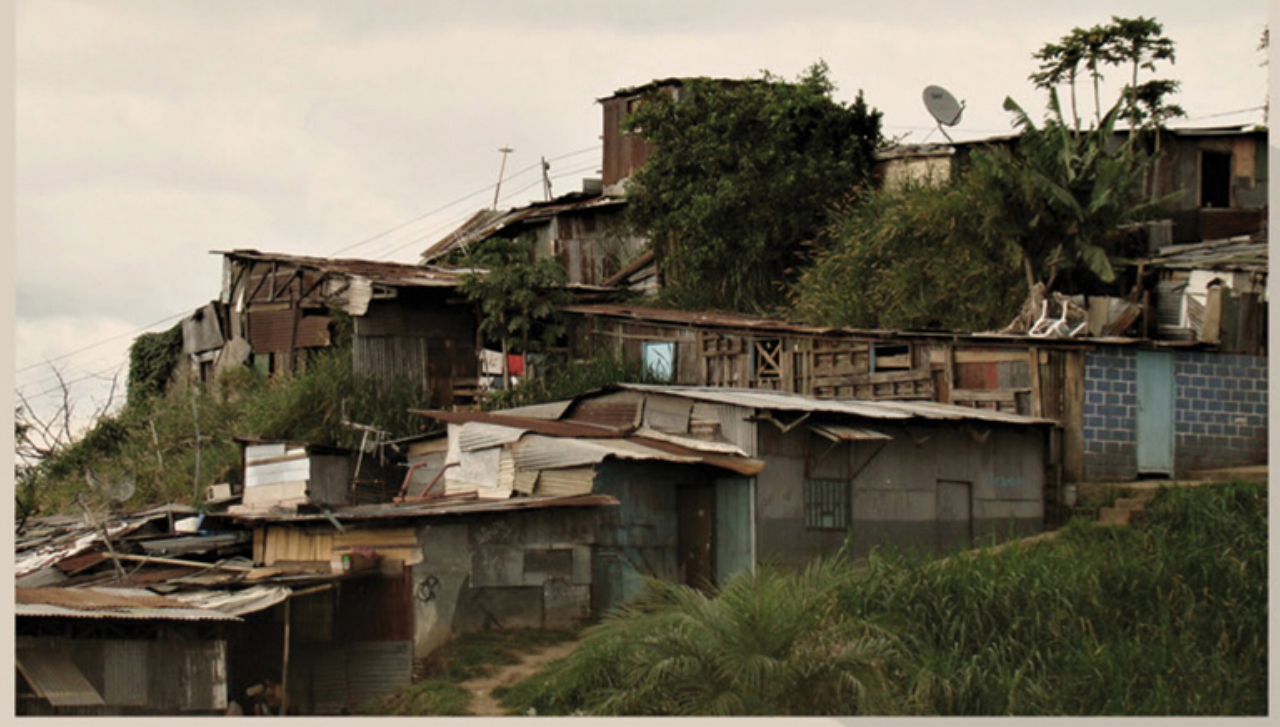

Centro de Investigaciones Históricas de América Central. Universidad de Costa Rica Julio-diciembre 2020

url: http://revistas.ucr.ac.cr/index.php/dialogos/index
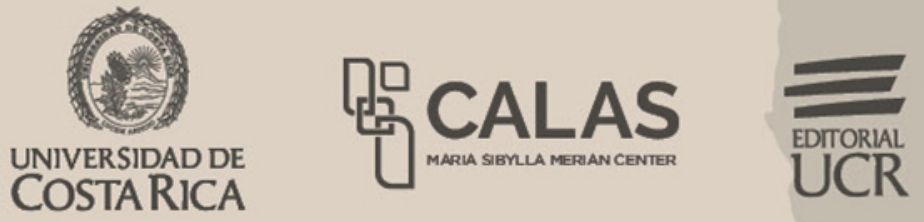


\title{
PUERTO RICO: EL COLAPSO DE UN PAÍS. UNA \\ REFLEXIÓN SOBRE EL DEBACLE DE LA INDUSTRIA \\ AZUCARERA, LAS PETROQUÍMICAS Y LA SECCIÓN 936.
}

Javier Alemán Iglesias

\begin{abstract}
Resumen
En este trabajo se tiene como objetivo central examinar las implicaciones socioeconómicas de la desintegración de la economía de Puerto Rico a partir de la segunda mitad del siglo XX usando como centro de estudio los casos de la industria azucarera, la industria de la petroquímica (CORCO) y las industrias bajo el sector de la sección 936. Los tres sectores fueron, en su momento, de apogeo y las actividades más importantes en la economía puertorriqueña durante el siglo XX; no obstante, los tres sectores fueron desplazados y arruinados por situaciones locales e internacionales, causando una de las crisis económicas más grandes de nuestra historia contemporánea.
\end{abstract}

Palabras clave: bancarrota, desempleo, crisis económica, pobreza, gobierno.

\section{PUERTO RICO: THE COLLAPSE OF A COUNTRY. AN INITIAL REFLECTION ON THE DEMISE OF THE SUGAR INDUSTRY, PETROCHEMICALS, AND SECTION 936.}

\begin{abstract}
In this work, the main objective is to examine the socio-economic implications of the disintegration of the economy of Puerto Rico from the second half of the twentieth century, using as a center of study the cases of the sugar industry, the petrochemical industry (CORCO), and the industries under the section of 936 sector. The three sectors were at their peak and the most important activities in the Puerto Rican economy during the twentieth century; However, the three sectors were displaced and ruined by local and international situations, causing one of the largest economic crises in our contemporary history.
\end{abstract}

Keywords: socio-environmental bankruptcy, unemployment, economic crisis, poverty, government. 


\section{INTRODUCCIÓN}

El 3 de mayo de 2017, a meses de haber comenzado como gobernador, Ricardo Rosselló Nevares declaró la quiebra de Puerto Rico (territorio bajo la jurisdicción de Estados Unidos desde 1898) con el fin de reestructurar la deuda pública que se estima en unos \$ 70000 millones de dólares. Según Rosselló, su decisión de acogerse al Título III por medio de la Ley federal estadounidense (Ley de Supervisión, Administración y Estabilidad Económica de Puerto Rico del 2016, conocida por sus siglas en inglés como Ley Promesa) fue para atender la crisis financiera de la isla luego que finalizara la moratoria que impedía a los acreedores acudir a los tribunales para reclamar el pago de la deuda al Estado Libre Asociado (El ELA es la relación jurídica entre Estados Unidos y Puerto Rico desde el 1952). Ese Puerto Rico en bancarrota a nivel general, según las estadísticas gubernamentales y reseñas de los periódicos locales, experimenta una tasa aproximada de pobreza del $45 \%$, un desempleo que fluctúa entre un $12 \%$ a un $18 \%$, una economía subterránea de 46\%, 1,4 millones de ciudadanos dependientes de asistencia social federal, un $92 \%$ de importación en los productos de consumo y de una reducción demográfica histórica a causa de la migración masiva hacia diversos estados de los Estados Unidos. Adicional, este triste cuadro se complicó cuatro meses después al enfrentarse al reto más grande de los últimos 80 años de su historia, que fue el azote del huracán María (Schwartz, 2018). Todos estos escenarios sin duda alguna incrementaron la desigualdad en gran parte de la isla. En fin, la economía del país había colapsado.

Ante esa nueva realidad y descartando las posturas oficiales de los partidos principales, como también ante la negatividad de auditar la deuda, es medular analizar las razones que provocaron tal difícil situación como también identificar quiénes son los responsables de generar la crisis económica puertorriqueña. Por tal razón, se tiene como objetivo central examinar las implicaciones socioeconómicas de la desintegración de la economía de Puerto Rico a partir de la segunda mitad del siglo XX, usando como centro de estudio los casos de la industria azucarera, la industria de la petroquímica (CORCO) y las industrias bajo el sector de la sección 936. Los tres sectores fueron en su momento de apogeo y de las actividades más importantes en la economía puertorriqueña durante el siglo XX; no obstante, los tres sectores fueron desplazados y arruinados por situaciones locales e internacionales, causando una de las crisis económicas más grandes de nuestra historia contemporánea.

\section{EL MONOCULTIVO PUERTORRIQUEÑO: AUGE Y DECADENCIA DE LA INDUSTRIA AZUCARERA}

El 25 de julio de 1898 las tropas militares estadounidenses desembarcaron en la isla de Puerto Rico como resultado de los planes de expansión por el Caribe del país invasor (García, 2013). Afirma Andrés Ramos Mattei (1974) en el artículo "Las inversiones norteamericanas en Puerto Rico" que, a dos semanas de la invasión, arribó en el 
municipio de Ponce un vapor (barco) de hombres de negocios interesados en conocer la disponibilidad de terrenos y de las ventajas comerciales e industriales que la isla podía ofrecerles. Ese grupo fue el primero de cientos que llegaron a suelo borincano durante la primera década del siglo XX en búsqueda de hacer riquezas en suelos tropicales. A partir de entonces, la industria azucarera puertorriqueña experimentó un auge sin precedentes, provocando la generalización del monocultivo insular. La razón principal para explicar dicho fenómeno fue la inclusión de la isla en el año 1901 como territorio doméstico (libre de impuestos y no extranjero) de los Estados Unidos dentro de los artículos del Código de Rentas Internas de la Ley Foraker (Ayala \& Bernabe, 2011). Tal medida facilitó la inversión de empresarios, comerciantes, banqueros y otros, atraídos por las sustanciales ganancias del mercado de la caña. Para el año 1910, ya se habían establecido más de 40 centrales azucareras de capital extranjero y nativo alrededor de toda la isla (García, 2013). Durante esos años, entre la segunda década y la mitad de la siguiente, la industria experimentó una bonanza sin precedentes a la cual se le llamó "la danza de los millones".

No obstante, entre finales de la década del veinte y principios de la próxima, la isla experimentó varios escenarios que comenzaron a perjudicar la industria del dulce. El 20 de septiembre de 1928, el huracán San Felipe azotó el país causando pérdidas significativas a la industria y sus cosechas. Como si fuera poco con el escenario descrito, ocurre la Gran depresión de 1929 que se extendió por gran parte de la década del treinta, aumentando la crisis de los agricultores y la producción en general por la falta de capital (Dietz, 2002). En medio de esa situación, el 26 de septiembre de 1932 nuevamente la isla es azotada por un huracán, San Ciprían, incrementado la crisis de la economía local, principalmente, en el sector de la industria del azúcar. Uno de los ejemplos que podemos destacar para describir tal situación y sus consecuencias fue la repentina quiebra de la corporación United Porto Rico Sugar Company (UPRSC), que era en ese momento una de las cuatro corporaciones ausentitas más grandes del país. Por otro lado, se puede señalar que a estos fenómenos se le añaden otros tres sucesos que sin duda alguna marcaron el inicio de la decadencia de la industria puertorriqueña. El primero es la aplicación de la Ley de Ajuste Agrícola en los EE. UU. y sus territorios en mayo de 1933 (Medina, 2015). Con esta ley en vigor, el Departamento de Agricultura de los Estados Unidos limitó la producción del azúcar y asignó cuotas anuales a los estados y territorios productores del dulce para evitar la caída de los precios, como resultado o consecuencia de la gran depresión de 1932. En el caso de Puerto Rico, se asignó 821000 toneladas, siendo esta cantidad menor a la esperada por los sectores productores que estimaban 1000000 toneladas de azúcar (Medina, 2015).

En segundo lugar, el gran debate que generó la discusión del Plan Chardón en el Senado que buscaba reformar la industria y sustituir una gran parte de las tierras dedicadas a la caña por cultivos de frutos menores. Finalmente, en tercer lugar, la creación de la Cooperativa Azucarera de Puerto Rico en 1937 a través de la PRRA que adquirió la Central Lafayette en Arroyo y la Central Los Caños en Arecibo, siendo el modelo la primera ocasión que el gobierno operó algunos aspectos de la industria (Tapia, 2014). Estos tres hechos sin duda alguna provocaron grandes cambios durante los años siguientes. 
Posteriormente, el ascenso del Partido Popular Democrático a partir de 1940 y su interés por desarrollar su proyecto de modernidad y de socializar la tierra entre los campesinos del país los llevó a implementar la Reforma Agraria por medio de la Ley de Tierras de marzo de 1941 (Nazario, 2014). Esta medida enfatizó la aplicación de la Ley de los 500 acres, ignorada desde el momento en que se firmó en 1900 por la oligarquía y los sectores latifundistas, y autorizó al gobierno de Puerto Rico a expropiar a toda persona o compañía el excedente a las 485.6 acres de terreno. Desde ese momento, el gobierno de Puerto Rico, dirigido por la Autoridad de Tierras, se convirtió en el máximo poseedor de la tierra en la isla y el sector agrícola más afectado durante el proceso fue la industria del azúcar. Gran parte de la tierra expropiada se destinó a proyectos sociales como la repartición de parcelas y creación de Fincas de Benéfico Proporcional, pero también a proyectos estratégicos como fueron los establecimientos de bases militares estadounidense (Ayala \& Bernabe, 2011).

Por otro lado, hay que señalar que durante esa década de la segunda posguerra ocurren cambios sustanciales como lo fue la Operación Manos a la Obra y el énfasis o el "afán" del Estado por modernizar, que sin duda alguna tuvo repercusiones directas en los espacios o terrenos cañeros desplazados para el uso de nuevas industrias, construcción de obras públicas o comerciales. Como consecuencia de dicha transformación junto a los altos costos de producción, la desfase entre la mecanización de la fábrica y las tecnologías del campo, el tiempo muerto y los bajos salarios, la inestabilidad del mercado y la demonización de la industria entre esa década del cuarenta hasta finales de los sesenta, cierran en la isla alrededor de 22 centrales azucareras (Zanetti, 2012). Una decena de municipios de diferentes regiones del país experimentaron cambios sustanciales en la economía a causa de la pérdida masiva de empleos directos e indirectos. Cabe destacar que muchos establecimientos de servicios, comidas y otros, se nutrían de las operaciones de los cañaverales, las colonias y de la factoría central, por lo cual se detuvo la actividad en la economía local.

Según las estadísticas sobre el grupo trabajador del Departamento del Trabajo, para el año 1950 hubo 33000 agricultores desempleados y de esa cantidad 24000 eran de la industria de la caña, representado un $73 \%$ de desempleo del sector agrícola. Para el 1964 se encontraban desempleados 19000 agricultores de los cuales 12000 eran de la industria azucarera, representando un 64\%, reflejando una disminución porcentual que se debió principalmente a que muchos de los agricultores comenzaron a trabajar en otras industrias y otros migraron hacia los Estados Unidos. Respecto a este último señalamiento, se encuentra que el gobierno de Puerto Rico, para contrarrestar la situación, utilizó el Negociado de Empleos y Migración del Departamento del Trabajo a través de un programa de relocalización de agricultores desempleados para que fueran a trabajar a los Estados Unidos. Según las estadísticas del Departamento, entre los años 1947 al 1961 migraron alrededor de 152253 agricultores y estos se establecieron en los estados de New Jersey, Pennsylvania, Connecticut, Massachusetts, Indiana, New York, Minnesota, Washington, Delaware, Michigan y Wisconsin, provocando tal migración un nuevo capítulo en nuestra historia contemporánea (Meléndez, 2017). 
Sin embargo, llama la atención que mientras el gobierno auspició la migración masiva de agricultores de caña a los Estados Unidos, así como el establecimiento de industrias con exenciones contributivas en zonas metropolitanas que facilitó la mudanza del campo a la cuidad; por otra parte, estaba subsidiando y alimentando una industria notablemente en decadencia. Es decir, el 25 de junio de 1969, el gobernador Luis A. Ferré firmó la Ley 24 que llevaba como título Programa de Rehabilitación de la Industria Azucarera (PRIA). Esta nueva ley asignó la cantidad de 100 millones de dólares para recuperar la producción del dulce, ya que, en los últimos años y principalmente la zafra de 1968, su producción nacional había descendido a 478 000 toneladas, siendo la más baja en casi medio siglo (Baralt, 2011). Además de esa cantidad de dinero, el gobierno operaba las Centrales Cambalache, Central Fajardo y la Central Juncos. Es decir, que la inversión del estado en la industria durante ese periodo era sustancial, teniendo en cuenta que ya la caña de azúcar había sido desplazada como el producto principal de exportación por la industria del atún.

Por otro lado, se ha encontrado que, de esos 100 millones, 61 fueron mal usados y peor aún, se endeudaron con 95 millones adicionales en el proceso de rehabilitar la industria. Los dos gobiernos siguientes, los de Rafael Hernández Colón y Carlos Romero Barceló continuaron la política de Ferré, sosteniendo la industria hasta principios de los 90, que, zafra a zafra, presentaba pérdidas millonarias para el país. De igual forma cabe señalar que bajo el primer cuatrienio de Hernández Colón (1973-1976) se creó, el 29 de marzo de 1973, la Corporación Azucarera de Puerto Rico (CAPR) con el propósito de centralizar las operaciones de la industria. Es ese proceso adquirieron la Central Plata, Central Roig, Central Coloso, Central Guánica, Central Aguirre, Central Eureka, Central Igualdad, Central Mercedita, las refinerías Roig, Mercedita e Igualdad por la cantidad de $\$ 32400000$ millones de dólares. A partir de entonces toda la industria estaba en manos de la Autoridad de Tierras, Agencia del Departamento de Agricultura de Puerto Rico. Al nacionalizar la empresa, la situación no mejoró y la nueva corporación tuvo que decidir, entre principios de la década de los 70 hasta mediados de los 90, cerrar 14 centrales adicionales, entre ellas algunas de las más importantes como eran la Guánica (1981), Aguirre (1990) y La Plata (1996). Tal situación empeoró las finanzas de la corporación y tuvo que desembolsar más de 15 millones durante el periodo señalado a los empleados y obreros cesanteados luego del cierre de las centrales.

Con esta situación y ante un estado crítico de la industria, la baja productividad, la escasez de mano de obra y el aumento de sus deudas, el exgobernador Pedro Roselló firmó la Ley 189 de septiembre 1996, en la cual liquidó y privatizó la Corporación Azucarera de Puerto Rico. En la transacción transfirió todos los activos y bienes muebles a la corporación Empresas Colonos de las Centrales Roig y Aguada. Esta empresa tuvo la misma suerte que la corporación anterior y continúo aumentando deudas zafra tras zafra hasta que decidieron finalizar las operaciones. La Central Roig finalizaría operaciones en 2001 y la Central Coloso en 2002, quedando en la calle alrededor de 3500 empleados. Con el cierre de ambas factorías, la industria azucarera puertorriqueña llegó a su fin, cerrando un capítulo de una historia económica que data sus inicios desde el siglo XVI. 
No obstante, con el cierre la triste situación no finalizó ahí. Al consultar varias vistas públicas del estado de Puerto Rico con la Autoridad de Tierras, se pudo encontrar un memorial explicativo sobre el presupuesto fiscal para el año 2017, en la cual dicha corporación alega tener una deuda de \$22 446680 de la Corporación Azucarera (eliminada en 1996). El balance pendiente se desglosaba en planes de retiro, convenios colectivos, acciones legales, obligaciones ambientales, entre otras, los cuales aún no han sido satisfechos por esperar alguna asignación de la Cámara Legislativa.

\section{LA ETAPA FINAL DE LA OPERACIÓN MANOS A LA OBRA: LA INDUSTRIA DE LAS PETROQUÍMICAS}

La isla de Puerto Rico, a partir del 1941, experimentó una serie de transformaciones políticas, económicas, sociales y culturales provocadas principalmente por los escenarios internacionales como fueron la Segunda Guerra Mundial, la expansión del capitalismo y el proceso de descolonización, entre otros. A nivel local, esas trasformaciones estuvieron dirigidas por el ascenso del Partido Popular Democrático y su líder Luis Muñoz Marín, que tenían como uno de sus objetivos mejorar las condiciones económicas y sociales de los puertorriqueños desarticulando el poder económico de los monopolios azucareros. La visión del PPD y su liderato causó una nueva etapa en la historia que facilitó la industrialización "parcial" del país. Esto ocurre al firmar en 1947 la Ley de Incentivos Industriales de Puerto Rico para empresas puertorriqueñas, estadounidenses e internacionales y al iniciar la promoción del sistema de incentivos contributivos federales que ya estaban disponibles para las empresas estadounidenses que hicieran negocios en Puerto Rico (y otras posesiones de EE.UU.). A ese proyecto se le llamó Operación Manos a la Obra que estuvo dirigido por Teodoro Moscoso, convirtiéndose en la prioridad principal de la Compañía de Fomento Industrial de Puerto Rico, la cual se había organizado en 1942 con el propósito de que el estado operara empresas (vidrio, botellas, fábricas de papel y cajas y una planta de cerámica y una fábrica de zapatos) y del Banco de Fomento de Puerto Rico (Dietz, 2002).

El objetivo del proyecto fue atraer masivamente industrias de capital externo (estadounidense) por medio de beneficios de exenciones contributivas a nivel local y federal sobre los ingresos y la propiedad. La Operación Manos a la Obra estuvo dividida en dos etapas esenciales y fueron muy distintas en sus actividades. Por ejemplo, la primera etapa se desarrolló desde 1947 hasta principios de 1960 en la cual las corporaciones (principalmente de los EE.UU.) que se establecían en la isla hacían uso intensivo de la mano de obra y requerían relativamente poco capital, por lo cual pagaban muy bajos salarios (Curet, 2003).

La segunda etapa, se desarrolló desde principios de los sesenta con el objetivo de aumentar los salarios de los puertorriqueños. Por lo tanto, la Compañía de Fomento se propuso extender y atraer industrias de capital intensivo como lo fue la industria de las petroquímicas, que usaban mano de obra diestra y semidiestra. 
La industria petroquímica nace en Puerto Rico como parte de la última etapa del programa Operación Manos a la Obra y, ya para el año 1953, se había construido la infraestructura para la producción de los derivados del petróleo entre los municipios de Peñuelas y Guayanilla. De todas las corporaciones que se establecieron durante ese periodo histórico, la que más se destacó en ese sector fue la compañía Commowelth Oil Refening Company, Inc., mejor conocida como CORCO (Baralt, 2011). La misma es una compañía organizada bajo las leyes del Estado Libre Asociado de Puerto Rico, que incluyó una refinería de petróleo con capacidad de 161 000 barriles diarios y ocho plantas petroquímicas. La refinería producía gasolina, queroseno, combustible para aviones a reacción, aceite diesel, aceites combustibles, propano, butano y otros productos derivados del petróleo.

El gobierno dirigió todos sus esfuerzos hacia esta industria y apostó que el crecimiento económico bajo este sector sería el futuro del país. Entre la década del 60 y principios de los 70, los ingresos de estas corporaciones representaban gran parte del ingreso bruto del país, desplazando la agricultura y otros sectores menores de manufacturas (Dietz, 2002). Además de inyectar la economía de Peñuelas y Guayanilla, otros municipios adyacentes se beneficiaron por los empleos creados directos e indirectos como también en el ofrecimiento de servicios. Sin embargo, la Guerra de Yom Kippur y el apoyo de los Estados Unidos y Occidente a Israel provocó la crisis del 1973 en el Medio Oriente. La consecuencia principal fue que los países Árabes Exportadores (OPEP) del petróleo bloquearon por un corto tiempo sus exportaciones para aumentar el precio del crudo. Los Estados Unidos, que era uno de los países que más dependía de las importaciones del oro negro, tuvieron que dejar de comprar petróleo para refinarlo en Puerto Rico a causa del embargo, provocando el fin de la bonanza. Para los Estados Unidos, traerlo a refinar a la isla le costaba mucho dinero. En 1982, CORCO se declaró en quiebra cerrando un capítulo más de la historia puertorriqueña.

\section{LA SECCIÓN 936: EL ÚLTIMO MODELO DE CRECIMIENTO ECONÓMICO, 1976-2006}

Ante la nueva crisis del petróleo, la salida inminente de ese negocio y la pérdida de capital, el gobierno logró que el Congreso firmara a la sección 936. Esta medida era un artículo del Código de Rentas Internas que determinaba los estatutos contributivos federales para las corporaciones de los Estados Unidos que se establecían en las "posesiones de los Estados Unidos" (Dietz, 2002). A partir de entonces miles de fábricas, farmacéuticas y empresas de tecnologías se establecieron en Puerto Rico en búsqueda de los beneficios que recibían bajo las 936, creando miles de empleos y causando un crecimiento económico inesperado (Baralt, 2011). No obstante, las exenciones contributivas crearon un gran malestar en diversos sectores de los Estados Unidos como el Departamento del Tesoro, el movimiento obrero y varios senadores del Congreso, ya que sostenían que Puerto Rico se había convertido en un paraíso fiscal para evadir contribuciones. 
La situación comenzó a generar presión y provocó varias enmiendas en las disposiciones del Código. Además, con el comienzo de la globalización y la desregulación de leyes laborales junto al tratado económico NAFTA, Puerto Rico dejó de ser atractivo para muchas empresas, ya que conseguían mejores beneficios en otros países del continente. Ante tal escenario, en 1996 el presidente Bill Clinton derogó la sección 936, permitiendo 10 años de gracia para aquellas empresas que quisieran permanecer con sus operaciones en la isla (Curet, 2003). A raíz de la eliminación, poco a poco decenas de fábricas fueron cerrando operaciones y cesanteando sin compensación alguna a los trabajadores, lo cual incrementó aceleradamente el camino a la gran crisis económica que estalló en el año 2005. Llama la atención que uno de los beneficios de las exenciones contributivas para estas industrias era que sus empresas podían repatriar el 90\% de sus ganancias y en el caso de las 936 (Muñiz, 2013), a excepción de los empleos de las farmacéuticas, todos los demás empleos eran salarios bajos sin ninguna otra compensación laboral. Por lo cual, aunque los incentivos de la sección 936 fueron importantes para el país (en su momento), se piensa que ese modelo no aportó al desarrollo en general del país ni tampoco al desarrollo humano.

\section{LAS IMPLICACIONES SOCIOECONÓMICAS DE LA DESINTEGRACIÓN DE LAS INDUSTRIAS}

Luego de analizar ese contexto, se pudo encontrar que luego de la desintegración de la industria azucarera, las petroquímicas y las fábricas de la sección 936 en la mayoría de los municipios que dependían de esas industrias, todos los indicadores económicos disminuyeron drásticamente y aumentaron con relación a la dependencia o ayuda del estado. En el caso del nivel de pobreza, todos esos municipios se encuentran entre 60,5 al 70,0\% bajo esa categoría, es decir, tres cuartas partes de la población. Cuando se coteja las estadísticas del Departamento de la Familia de la asistencia nutricional o mejor conocido como el PAN, se identifica que los municipios de San Sebastián, Guánica, Salinas y gran parte del litoral sureste son los mayores beneficiarios de dicha ayuda federal (Colón, 2011). Respecto a la deuda municipal, el $92 \%$ de esos municipios llevan más de 10 años con déficit fiscal que fluctúa entre 4 a 7 millones de dólares anuales. Por otro lado, cuando se mira el desempleo por municipio, al menos las estadísticas de los últimos 40 años del Departamento del Trabajo (en la serie histórica), se encuentra que los municipios más afectados por la inactividad laboral son los que fueron agrícolas, principalmente los de caña, aunque también en la última década incrementó el número en las regiones que dependían de las empresas de la 936. Otro aspecto significativo es la disminución demográfica en estas localidades, pues miles de trabajadores y sus generaciones siguientes continuaron con la tendencia de buscar en zonas más desarrolladas, o la zona "metro", algún empleo como también migrando a los Estados Unidos, provocando el aumento de diversos lugares abandonados y menos recaudos para el estado o el municipio (Meléndez \& Vargas, 2014). 
En el caso concreto del cierre de la Central Guánica en 1981, se identifica que causó el desempleo que ascendió a un 50\% y el poblado de Ensenada se convirtió en uno de los más necesitados del suroeste de la isla (Ramos, 2002). El cambio físico, económico, social, y estético luego del cierre, fue notable como también el aumento del crimen y el uso de drogas según muestran los índices del Departamento de la Policía. La mayoría de las estructuras usadas por la Central fueron abandonadas, empobreciendo aún más el poblado. Todos esos efectos son palpables en lugares cañeros e industriales, donde no se pudo implementar alguna estrategia transicional para minimizar el impacto de la desaparición de las industrias señaladas.

\section{CONSIDERACIONES FINALES}

Para finalizar se sugiere que la falta de un modelo económico transitorio dirigido por el estado y el capital privado en los inicios de la globalización durante el proceso de la desaparición de la industria azucarera, las petroquímicas y los incentivos de la sección 936 provocó en las regiones o sectores donde se llevaban a cabo esas actividades económicas un sinnúmero de complicaciones socioeconómicas y ambientales. Tal situación causó en la mayoría de los municipios una gran recesión a raíz de los miles de empleos que se perdieron con el fin de las industrias, incrementando el deterioro y el abandono en los barrios y lugares adyacentes. Por otro lado, la falta de trabajo obligó a muchos agricultores, obreros y técnicos a relocalizarse a otros municipios de la isla donde se desarrollaba el modelo en los empleos de servicio y turismo, y otros a migrar a los EE.UU. Los que permanecieron en los lugares mencionados poco a poco comenzaron a depender de las ayudas sociales federales o la dependencia del estado para obtener el sustento diario, lo cual causó un estancamiento económico en la región y aceleró el deterioro físico y humano en dichas localidades, reflejando notablemente una mayor pobreza y desigualdad. Peor aún, el gobierno, en el afán de eliminar el monocultivo e industrializar el país, extinguió la agricultura y dejó sin la posibilidad de convertir el país en autosuficiente en cuanto a alimentación al no considerar que el futuro era (o es) diversificar la producción agrícola. Además, cerró cualquier plan futuro para que los puertorriqueños tuvieran un transporte colectivo al eliminar el ferrocarril e introducir los vehículos de motor.

Por otra parte, el negocio del azúcar provocó un impacto ambiental significativo ya que durante el proceso de producción se depositaron desperdicios en los ríos cercanos a sus fábricas. Adicional, luego de su cierre tampoco hubo una limpieza de sus ruinas o lugares adyacentes, incrementando la contaminación en esos lugares. Muchos de estos son usados clandestinamente como vertederos de basura o viviendas ilegales de usuarios de drogas. En el caso de las petroquímicas, que principalmente operaron en los municipios de Guayanilla y Peñuelas, consta que sus tierras, aires y acuíferos siguen contaminados con químicos como benceno, tolueno, benceno etílico, xileno, naftalina, entre muchos otros. En el caso del aire de la zona, se encuentran muchísimas partículas finas con metales pesados que pueden causar 
(o causan) enfermedades respiratorias, cardiovasculares y hasta cáncer. Finalmente, en el caso de empresas de la sección 936, principalmente las empresas farmacéuticas, es de conocimiento público que los niveles de contaminación de los terrenos y corrientes de aguas son altísimos a causa de los desperdicios tóxicos, principalmente carcinógenos, depositados ilegalmente por estas empresas como también al aire por las partículas de químicos que se desprenden del proceso de fabricación.

\section{REFERENCIAS}

Ayala, C. y Bernabe, R. (2011). Puerto Rico en el siglo americano: su historia desde 1898. San Juan: Ediciones Callejón.

Baralt, G. (2011). La Gran tarea: la obra de gobierno de Luis A. Ferré, 1969-1972.San Juan: Ediciones Puerto.

Colón Reyes, L. (2011). Sobrevivencia, pobreza y “mantengo”. La politica asistencialista estadounidense en Puerto Rico: el PAN y el TANF. San Juan: Ediciones Callejón.

Curet Cuevas, E. (2003). Economía Política de Puerto Rico: 1950 a 2000. San Juan: Ediciones M.A.C.

Dietz, J. (2002). Historia Económica de Puerto Rico. Río Piedras: Ediciones Huracán.

García Muñiz, H. (2013). De la Central Guánica a la Central Romana: La South Porto Sugar Company en Puerto Rico y la República Dominicana, 1900-1921. Santo Domingo: Academia Dominicana de la Historia.

Meléndez, E. (2017). Sponsored Migration. The Estate and Puerto Rican postwar migration to the United States. Columbus: The Ohio State University Press.

Medina, H. (2015). La Guerra del azúcar. San Juan: Publicaciones Gaviota.

Meléndez, E. y Vargas Ramos, C. (2014). Puerto Ricans at The Dawn of New Millennium. New York: Center for Puerto Rican Studies.

Muñiz Velera, M. (2013). Adiós a la economía. San Juan: Ediciones Callejón.

Nazario Velasco, R. (2014). El paisaje y el poder: La tierra en el tiempo de Luis Muñoz Marín. San Juan: Ediciones Callejón.

Ramos Mattei, A. (1974). Las inversiones norteamericanas en Puerto Rico y la Ley Foraker, 1898 1900. Caribbean Studies, 14(3), 53-70.

Ramos, M. (2002). La Muerte de un gigante. San Juan: Editorial Plaza Mayor.

Schwartz, Stuart B. (2018). Mar de Tormentas: una historia de los huracanes en el Gran Caribe desde Colón hasta María. San Juan: Ediciones Puerto.

Tapia Ríos, G. (2014). La Central Lafayette: riqueza, desarrollo y político en el sureste de Puerto Rico. San Juan: Ediciones Magna Cultura.

Zanetti, O. (2012). Esplendor y decadencia del azúcar en las Antillas hispanas. La Habana: Ciencias Sociales. 